\title{
O ESTILO DE VIDA DO CLIENTE COM HIPERTENSÃO ARTERIAL E O CUIDADO COM A SAÚDE
}

\author{
The Arterial Hypertension Patient's Life Style \\ and the Health Care \\ El Estilo de Vida del Cliente con Hipertensión y \\ el Cuidado con la Salud
}

Enéas Rangel Teixeira
Juliana da Costa e Silva

Alinny Rodrigues Lamas

Ronivaldo Menegussi de Matos

\begin{abstract}
Resumo
Esta pesquisa tem como objetivo descrever os componentes da história de vida do sujeito que influenciam o cuidado com a saúde. A pesquisa é quantitativa, e sua amostragem é de 220 clientes adultos e idosos de uma Unidade de Saúde de Niterói-RJ. Quatro temáticas foram analisadas: características do grupo, hábitos de vida, comportamentos aditivos e estado emocional. $50 \%$ dos clientes tinham entre 50 e 70 anos; $38 \%$, mais de 70 anos. $81 \%$ eram do sexo feminino. $65 \%$ possuíam o $1^{\circ}$ grau incompleto. $54 \%$ não praticavam atividades físicas. $46 \%$ dos sujeitos já fizeram uso de tabaco. $37 \%$ fizeram uso de bebida alcoólica. $41 \%$ dos sujeitos consideravam-se tranqüilos, e $59 \%$ apresentavam transtornos emocionais. $27 \%$ não realizavam atividades de lazer. 0 estilo de vida é fundamental para a promoção e manutenção da qualidade de saúde, e isso requer estratégias de trabalhos adequadas aos aspectos psicossociais do sujeito.
\end{abstract}

Palavras-chave: Estilo de Vida. Atenção à Saúde. Pressão Arterial. Hipertensão. Enfermagem.

\begin{abstract}
The goal is: to research the life history components' patient that influences the care with health. This is a quantitative research. The sample was 220 adult and elder patients in a Health Unit at Niterói-RJ. Four themes were analyzed: group feature, life habit, additional behavior and emotional state. $50 \%$ of the patients were from 50 until 70 years old, $38 \%$ were more than 70 years old, $81 \%$ were feminine sex, $65 \%$ have been not completed the first degree, $54 \%$ don't practice exercises, $46 \%$ have already used tobacco, $37 \%$ have been used booze, $41 \%$ profess to be peaceful, $59 \%$ reports emotional disarrays and $27 \%$ don't practice leisure activities. The life style is fundamental to promote and maintain health quality and it necessitate job strategy in keeping with patient psychosocial aspects.
\end{abstract}

\section{Resumen}

Esta pesquisa ten como objectivos describir los componentes de la história de vida del sujeito que influencian el cuidado con la salud. La investigación és cuantitativa. La muestra fué 220 clientes adultos y ancianos de una Unidad de Salud de Niterói-RJ. Cuatro temáticas fueron analisadas: característica del grupo, hábitos de vida, comportamientos adictivos y estado emocional. $50 \%$ de los clientes tenían entre 50 a 70 años, $38 \%$ con más de 70 años. $81 \%$ era del sexo femenino. $65 \%$ tenían el $1^{\circ}$ grado incompleto. $54 \%$ no praticaban actividades físicas. $46 \%$ de los sujeitos habían usado tabaco. $37 \%$ usó bebida alcohólica. $41 \%$ de los sujetos considéranse tranquilos y $59 \%$ presentan transtornos emocionales. $27 \%$ no practican actividades de lazer. El estilo de vida és fundamental para la promoción y manutención de la cualidad de salud y eso requiere estrategias de trabajo adecuadas aos aspectos psicosociales del sujeto.

Keywords: Life Style. Health Care. Blood Pressure. Palabras clave: Estilo de Vida. Atención a la Salud. Presión Hypertesion. Nursing. 


\section{CONSIDERAÇÕES INICIAIS}

Este trabalho emerge de nosso cotidiano, que lida com as formas de vidas de clientes numa unidade básica de saúde. Desse modo, temos o intuito de conhecer melhor o estilo de vida do usuário, relacionado-o com o cuidado em saúde. 0 conceito de estilo de vida passou a ser adotado para explicar a ocorrência de agravos à saúde, nos quais o modo de vida do sujeito tem um papel importante. Assim para determinados autores, a doença pode resultar de formas pouco "saudáveis" de viver ${ }^{1}$. Aparentemente isso pode parecer óbvio, numa perspectiva de causa e efeito, sem levar em consideração os vieses do contexto da saúde.

Entretanto, as condições de vida urbana contribuem sensivelmente para o surgimento do mal-estar social e dos agravos crônicos e degenerativos, e fazem das doenças cardiovasculares um problema da modernidade. Coadjuvantes dessa situação do contexto, os aspectos subjetivos delineiam um modo de 0 sujeito lidar com as tensões do cotidiano ${ }^{2}$.

Para compreender o estilo de vida, é importante entendê-lo dentro de um contexto social da saúde, deslocando-se do modelo centrado na doença. 0 novo paradigma da saúde - que contempla a complexidade, a determinação social e a perspectiva holística do ser - vem sendo cada vez mais preconizado nas conferências nacionais e internacionais de saúde ${ }^{3}$.

0 conceito de campo na saúde abarca: a biologia humana, a qual abrange a herança genética e os processos vitais; o meio ambiente, que inclui o território geográfico, como algo vivo e dinâmico, envolvendo a ecologia e as relações existentes; o estilo de vida, do qual resultam decisões pessoais, lazer, cuidados com o corpo, trabalho, filosofia de vida, comportamentos aditivos; e a organização da assistência à saúde, que compreende a assistência e os serviços de saúde, nos diferentes níveis de atenção em saúde. ${ }^{4}$

Enfim, diante dessas considerações, temos como objetivos: levantar os componentes do estilo de vida do sujeito que influenciam o cuidado com a saúde; descrever como o aspecto emocional do sujeito se relaciona com os hábitos de vida e analisar os conteúdos emergidos de modo contextualizado.

\section{ESTILO DE VIDA E HIPERTENSÃO ARTERIAL}

0 estilo de vida é compreendido como um modo de viver que conduz à maneira de ser do sujeito, aos hábitos e suas expressões. A forma de vida da pessoa varia de acordo com o grupo social e cultural em que a mesma se encontra inserida. A decisão do indivíduo para manter uma forma peculiar de vida envolve os aspectos externos e os processos mentais. Num determinado sentido, os chamados fatores de riscos, como tabagismo, etilismo, alimentação inadequada, sedentarismo e estresse, são formas adaptativas do sujeito diante das tensões do cotidiano ${ }^{5}$.

A hipertensão arterial é uma doença atual, resultante das condições de vida do homem moderno, que expressa sua forma de viver e as contradições sociais existentes. Esse agravo representa um alto custo social na saúde, por causar enfermidades secundárias de peso, tais como: doença cerebrovasculares, transtornos cardíacos e complicações renais ${ }^{6}$, que podem levar à incapacidade e à morbidade. A hipertensão arterial é uma doença que acomete $28,5 \%$ dos brasileiros, e que, se não for tratada e controlada, resulta em graves complicações ${ }^{7}$.

Estudos quantitativos atuais contabilizam que há 600 milhões de hipertensos no mundo. Calcula-se que essa doença causa a morte de 7,1 milhões de pessoas, equivalente a $13 \%$ do total de óbitos. No Brasil, de acordo com o Sétimo Consenso Brasileiro para o Tratamento da Hipertensão Arterial - JAMA, cerca de 17 milhões de brasileiros são hipertensos, e a maior parte são pessoas em idade economicamente ativa, aumentando consideravelmente os custos sociais por invalidez e absenteísmo ao trabalho ${ }^{8,9}$.

Entende-se que o estilo de vida envolve a subjetividade do sujeito em seu contexto social, de modo que a objetivação da saúde e ou da doença tem uma dimensão psicossomática ${ }^{10}$, que não pode ser ignorada nas intervenções de saúde. Desse modo, as doenças, além dos aspectos sociais e fisiopatológicos, possuem associação com a emoção, na qual as condições corporais afetam a mente e vice-versa, num processo complexo e relacionado com o meio.

A manifestação da doença não pode ser explicada apenas por relação de causa e efeito, mas pelo contexto social e pelo estilo de vida que o indivíduo, como ser biológico e psicológico, se encontra inserido.

\section{CAMINHO METODOLÓGICO}

Este estudo é de natureza quantitativa e utilizou o método de estatística descritiva, de freqüência simples e percentual. 0 universo considerado abrange 1.126 clientes cadastrados no Programa de Hipertensão Arterial e Diabetes (HIPERDIA) do Ministério da Saúde na Policlínica Sérgio Arouca da Fundação Municipal de Saúde de Niterói-RJ.

A amostra foi de 220 clientes, que corresponde a $19,5 \%$ dos cadastrados no programa HIPERDIA, e foi 
escolhida de forma aleatória dentro da população do estudo, enquanto aguardava consulta dos profissionais de saúde.

0 dados foram coletados por meio de questionário fechado que contemplou os aspectos mesuráveis do estilo de vida, tais como idade, sexo, escolaridade, profissão, tipos de hábitos (atividade física, dieta), estado emocional e comportamentos aditivos (etilismo e tabagismo).

A coleta de dados ocorreu no período de fevereiro a novembro de 2005. A participação dos depoentes realizou-se mediante a assinatura do termo de consentimento livre e esclarecido, sendo assegurado aos participantes sigilo quanto às informações prestadas, de acordo com a Resolução no 196/96, do Conselho Nacional de Saúde. A pesquisa foi aprovada pelo comitê de ética da Universidade.

Os resultados foram tabulados e organizados em quatro temas: Característica do Grupo Pesquisado, Hábitos de Vida, Comportamentos Aditivos e Estado Emocional. Posteriormente, essas categorias foram discutidas e submetidas à discussão com auxílio de literatura acerca do assunto.

\section{RESULTADOS}

\section{Características do grupo pesquisado}

Os sujeitos da pesquisa são em sua maioria adultos e idosos, com predomínio da faixa etária de 50 a 70 anos, correspondendo a $50 \%$ do total desses depoentes. $38 \%$ dos depoentes tinham idade superior a 70 anos, e apenas 12\%, idade entre 30 e 50 anos.

Apesar de a maioria dos clientes hipertensos ser idosa, não se pode atrelar a doença de modo estrito ao processo do envelhecimento, pois essa envolve um conjunto de componentes que geram os agravos à saúde com o passar dos anos, tais como o estilo de vida, condições psíquicas, sociais e ambientais.

Entretanto, determinados autores consideram que o envelhecimento é um fator de risco importante no surgimento da hipertensão arterial, em decorrência das alterações anatômicas e fisiológicas decorrentes da idade ${ }^{11}$.

0 processo de envelhecimento da população no Brasil está ocorrendo de modo acelerado, e, associadamente, a crise econômica, as desigualdades e os problemas sociais contribuem para impedir que o idoso tenha uma vida saudável e satisfatória. Neste sentido, as doenças crônicas são responsáveis pelas principais causas de morte entre os idosos ${ }^{12}$.
Ao correlacionar idade e sexo, percebe-se que a maioria, $81 \%$ dos depoentes dessa pesquisa, pertence ao sexo feminino, talvez em virtude da sua maior disponibilidade para comparecer ao ambulatório, uma vez que em nossa sociedade ainda predominam as atividades de trabalho relacionadas ao gênero. Assim, cabe ao homem trabalhar para o sustento da família e, à mulher, realizar as atividades do lar, ficando esta, de certa forma, mais disponível para o acompanhamento na unidade de saúde.

Todavia, há também maior longevidade das mulheres em relação aos homens, pois dados oficiais mostram que as brasileiras vivem cinco anos mais do que os homens. As perspectivas são de que esta diferença continue aumentando, isto porque as taxas de mortalidade masculinas vêm se intensificando, principalmente por causas violentas, especialmente nos estados da região Sudeste ${ }^{12}$.

A maior expectativa de vida das mulheres, no entanto, não corresponde à melhor qualidade de vida. Os problemas sociais, econômicos e de saúde dos idosos homens, são, em grande parte, também os das mulheres idosas $^{12}$. De modo concomitante, a anciã continua realizando trabalhos domésticos e educando as crianças, sendo, não raramente, exploradas pela família.

Em relação à profissão, a maioria dos sujeitos é aposentada (39\%) pelo o tempo de serviço e em razão de doença incapacitante. Vale ressaltar que a maioria realizava trabalhos que requeriam habilidades físicas. Eram, por exemplo: construtores civis, lavradores, empregadas domésticas etc. Existe uma relação entre a profissão/ocupação e a elevação da pressão arterial, sendo que os índices mais baixos de pressão arterial ocorrem no grupo socialmente favorecido ${ }^{11}$.

Quanto à escolaridade, 65\% dos depoentes estudaram até 0 ensino fundamental, $24 \%$ até 0 ensino médio, e apenas $5 \%$ possuem ensino superior. Observase que o aumento da escolaridade é inversamente proporcional à porcentagem de hipertensos. Constatase uma tendência na queda da média da pressão arterial à medida que o grau de educação aumenta. Isto pode interferir diretamente na compreensão das orientações necessárias ao tratamento ${ }^{7}$, tendo em vista que a realização do autocuidado, no caso de cliente hipertenso, requer certo conhecimento. Entretanto, existe na população brasileira um predomínio de analfabetos funcionais, aqueles que não conseguem ler e escrever plenamente, de modo que educação e saúde andam juntas de certa forma e expressam as contradições sociais. 


\section{Hábitos de Vida}

0 objetivo do cuidado da pessoa com hipertensão arterial $^{13}$ é evitar a morte e as complicações, por meio do controle da pressão arterial, permitindo a melhoria da qualidade de vida. Portanto, é imprescindível que o sujeito compreenda o processo da doença e participe da mudança do estilo de vida por meio das atividades de educação em saúde.

Em relação à alimentação, a questão do cloreto de sódio foi uma constante, tanto por parte da exigência dos profissionais de saúde como pela preocupação dos clientes. Assim, $64 \%$ dos sujeitos responderam que sua dieta é preparada com pouco sal, e apenas $8 \%$ responderam que utilizavam dieta hipersódica. Confrontando com outra pesquisa ${ }^{11}$, observa-se certa similaridade; $74,3 \%$ dos depoentes desse outro estudo afirmam preparar o alimento com baixa quantidade de sal. Todavia, apesar de a maioria dos clientes ter apresentado esse resultado, surgem dúvidas em relação à quantidade ideal do consumo de sódio, pois cada sujeito cria o seu próprio critério. A orientação quanto ao consumo da quantidade ideal de sal é extremamente importante, pois a dieta hipersódica pode elevar a pressão arterial, oferecendo riscos à saúde. Todavia, se faz necessário encontrar formas criativas e prazerosas na alimentação, pois o gosto e o bem-estar são importantes para a saúde.

Sobre a ingestão de alimentos com lipídio, $27 \%$ responderam que não ingerem alimentos gordurosos, $51 \%$ responderam que ingerem pouco, $24 \%$, razoavelmente, e apenas $8 \%$ declararam ingerir grande quantidade. Sabe-se que a ingestão de gorduras em indivíduos portadores de hipertensão arterial deve ser reduzida, pois há aumento das complicações, como doenças coronárias e obesidade.

Percebe-se que os aspectos culturais aparecem no estilo de vida do sujeito, de modo que não compete a nós realizar uma educação que vise a excluir esses valores, mas trabalhar com eles de modo contextualizado, interativo e criativo no processo de cuidar.

No que diz respeito à realização de atividade física, $54 \%$ não a praticam ou a fazem raramente, e $46 \%$ realizam exercícios de modo regular. Ocorre uma relação inversa entre o grau de atividade física e a incidência de hipertensão; ou seja, à medida que se intensifica a atividade física, tem-se como efeito a redução dos níveis pressóricos, entre outros benefícios para o sujeito. Assim, o exercício físico tem importante papel como elemento não medicamentoso para o seu controle ou como adjuvante ao tratamento farmacológico ${ }^{14: 9}$

A atitude terapêutica do cuidado procura encorajar o cliente a rever seu estilo de vida, sua percepção e maneiras de se relacionar em seu contexto social, de modo a encontrar recursos e novas maneiras de viver com satisfação. No entanto, é necessário entender que não se trata de um processo fácil e linear, pois requer desejo e motivação para mudança.

Esse processo, para Potter ${ }^{15}$, envolve um movimento que se efetua através de cinco estágios: a pré-contemplação, entendida como a ausência da intenção de mudar; a contemplação, quando se considera 0 ato de mudar iminente; a preparação, na qual se realizam pequenos esforços para se modificar; a ação, na qual o sujeito está ativamente engajado nas estratégias para a mudança de comportamento; e, finalmente, a manutenção de um comportamento modificado.

No entanto, é preciso ter o entendimento de que outros aspectos também são decisivos nesse processo de mudança do autocuidado, como a oferta de serviços de saúde à população, a condição socioeconômica e as desigualdades sociais, que agem coletivamente na saúde da população.

\section{Comportamentos Aditivos}

Os comportamentos aditivos são também chamados de compulsivos, entendidos como hábitos ou atitudes repetitivas, que se apresentam de forma freqüente e excessiva. Tais hábitos propiciam gratificação emocional, de modo que o indivíduo busca alívio da angústia através de substitutos que proporcionam satisfação. Como exemplos de comportamentos aditivos, podemos citar o uso abusivo do álcool e do fumo, que acarretam conseqüências negativas à saúde, como o agravamento no controle da pressão arterial $^{16}$. Nessa perspectiva, enfocamos nesse estudo o tabagismo e o etilismo.

Assim, $46 \%$ dos indivíduos declararam-se tabagistas ou fumaram em determinados momentos da vida. Destes, $47 \%$ disseram consumir menos de um maço/ dia, $36 \%$ consumiam um maço/dia, e $17 \%$, de dois a quatro maços de cigarro por dia.

Apesar de estudos epidemiológicos não apontarem associação direta entre o fumo e a hipertensão arterial, esta mostra correlação estreita com a arteriosclerose que seria um fator agravante da doença hipertensiva. Isto se explica, pois, além do tabaco comprometer a 
integridade dos vasos sanguíneos, a nicotina, substância encontrada no fumo, produz a liberação de catecolaminas, que aumentam a freqüência cardíaca, a resistência periférica e, conseqüentemente, a pressão arterial. Portanto, os fumantes têm mais chance de apresentar formas malignas da doença hipertensiva, que acarretam incidência elevada de morte súbita ${ }^{17}$.

Ao contrário do tabagismo, o etilismo tem demonstrado íntima ligação com a hipertensão arterial. 0 aumento das taxas de álcool no sangue eleva a pressão arterial de modo lento e progressivo, na proporção de $2 \mathrm{mmHg}$ para cada $30 \mathrm{~mL}$ de álcool etílico ingeridos diariamente, sendo que, quando este é suspenso, as cifras se revertem ${ }^{18}$. Nesse estudo, $63 \%$ dos clientes disseram que não fazem uso de nenhum tipo de bebida alcoólica, $34 \%$ bebem eventualmente, e apenas $3 \%$, freqüentemente ${ }^{18}$.

Contudo, os dados foram significativos, demonstrando que tanto o tabagismo como o etilismo fizeram ou fazem parte dos hábitos de nossos clientes. Isto não é de se estranhar, pois o uso dessas substâncias é lícito em nossa cultura e, amiúde, estimulado. Além do mais, é importante compreender os aspectos culturais e psíquicos envolvidos nesse processo e não realizarmos juízos negativos de valores, que podem gerar efeitos negativos no cuidado da pessoa.

\section{Estado Emocional}

Os clientes foram abordados quanto a sua opinião sobre o seu estado emocional mais freqüente. Cerca de $41 \%$ dos entrevistados consideram-se estáveis em termos emocionais, enquanto a maioria, $59 \%$, apresenta algum tipo de transtorno emocional $(25 \%$ estressados, $23 \%$ ansiosos, $6 \%$ deprimidos e $3 \%$ outros), sendo que $27 \%$ deles não desenvolvem atividades de lazer que poderiam contribuir para amenizar tal comportamento.

Estudos sobre os aspectos psicológicos da hipertensão essencial ${ }^{19}$ evidenciam que a dificuldade de expressar os sentimentos hostis contribui para gerar o quadro clínico da doença. Assim, o estresse e a ansiedade são aspectos emocionais que podem induzir estilos de vida com a qualidade de saúde comprometida, como no caso da hipertensão arterial e suas complicações ${ }^{20}$.

0 trabalho de Machado, Stipp e Leite ${ }^{21}$ evidenciou que $71,9 \%$ dos seus entrevistados se consideraram constantemente submetidos a situações de estresse, o que justificaria o aumento pressórico, embora ainda não esteja suficientemente comprovado o papel do estresse na gênese da hipertensão.

Quanto à influência do estado emocional na pressão arterial, $81 \%$ dos sujeitos desse estudo responderam que há influência, 10\% acharam que às vezes ele pode influenciar, e 9\% acreditam que não influencia.

Esses dados evidenciaram o quanto o estado emocional é importante no bem-estar do sujeito. Diante de fortes emoções, o indivíduo desencadeia inúmeras reações somáticas e comportamentais, como, por exemplo, as alterações cardiovasculares. Em razão disso, sintomas cardíacos podem configurar-se como reflexo ou expressão de fortes tendências agressivas ou reprimidas. Podemos exemplificar como os sentimentos de hostilidade intensa possibilitam a elevação da pressão arterial. A constante tensão e contenção de emoções, que são mecanismos adaptativos distorcidos, podem manter a hipertensão prolongada, de maneira a dificultar o seu controle ${ }^{11}$.

Outro aspecto significativo relacionado à vida emocional do sujeito se refere à atividade sexual. A maioria colocou que a prática sexual é inexistente ou insuficiente. Nesse sentido, cerca de $45 \%$ não realizam atividade sexual, e $37 \%$ realizam raramente, sendo que apenas $34 \%$ consideram a relação satisfatória, $45 \%$ consideram indiferente, e $6 \%$, insatisfatória. Vale ressaltar que isso expressa uma realidade do universo feminino idoso, haja vista que $80,5 \%$ são constituídos de mulheres com idade acima de 60 anos.

Sabe-se atualmente, por meio do conhecimento da psicologia e da sexologia, que, quando o sujeito se encontra ajustado na sua vida sexual, com seu prazer e desejo, ocorre um reflexo expressivo em seu bemestar. Muitos problemas psicossomáticos são gerados por bloqueios da energia sexual22. Desse modo, é importante que nós profissionais de saúde estejamos atentos a essa dimensão da vida do sujeito, desenvolvendo conhecimentos e habilidades.

Portanto, é necessário compreender a dimensão psíquica no cuidado, pois as ações terapêuticas não requerem somente administração de medicamentos e ações prescritivas. Nesse sentido, o estudo da psicossomática contribui para o processo da dinamização e efetivação do cuidado de enfermagem, levando em consideração os aspectos emocionais do indivíduo, que abarcam sua condição de saúde e doença.

\section{CONSIDERAÇÕES FINAIS}

Realizamos um levantamento de determinados aspectos do estilo de vida do cliente, que envolvem 0 
cuidado e o controle da hipertensão arterial. Percebese que os hábitos enfocados aqui estão relacionados às práticas descritas na literatura de educação em saúde, quanto à alimentação, exercícios físicos, lazer, comportamentos aditivos, aspectos emocionais, entre outros. Desse modo, existe uma relação entre o cuidado na saúde e o estilo de vida. Todavia, é necessário compreender o estilo de vida levando em consideração o contexto social e os aspectos subjetivos, de modo a favorecer uma atitude crítica e sensível nas práticas de saúde ${ }^{23}$.

Percebe-se que os aspectos emocionais motivam a mudança, pois envolve o desejo e a satisfação pela vida. Os problemas mentais e a presença de poucas atividades prazerosas indicam um comprometimento com a satisfação do viver, coadjuvante às condições do contexto social, gerador de contradições. Por sua vez, o surgimento da doença expressa os efeitos de conflitos, que se manifestam na realidade biofísica do sujeito.

É importante entender os hábitos de vida dos sujeitos como formas adaptativas dos mesmos diante das tensões da vida. Neste sentido, a mudança do sujeito não passa por um processo linear e impositivo

\section{Referências}

1. Lessa I. 0 adulto brasileiro e as doenças da modernidade: epidemiologia das doenças crônicas não-transmissíveis. São Paulo (SP): HUCITEC; 1998.

2. Ballone GJ. Psicossomática e hipertensão arterial. PsiqWeb [on line]. 2003; [citado outubro de 2004];. Disponível em: http:// gballone.sites.uol.com.br/psicossomatica/cardiologia5.html

3. Ministério da Saúde (BR). Secretaria de Políticas de Saúde. Projeto promoção da saúde: as cartas da promoção da saúde. Brasilia (DF); 2002.

4. Scilar M. Do mágico ao social. Porto Alegre (RS): L \& PM; 1987.

5. Martins IS et al. Doenças cardiovasculares ateroscleróticas, dislipidemias, hipertensão, obesidade e diabetes mellitus em população da área metropolitana da região sudeste do Brasil. Rev Saúde Pública 1997 out; 31(5): 466-471.

6. Sociedade Brasileira de Hipertensão-SBH. IV Diretrizes Brasileiras de Hipertensão, 4 Campos do Jordão (SP) 2002 jun; [citado nov 2004]. Disponível em: http://www.sbh.org.br/documentos/index.asp

7. Simonetti JP, Batista L, Carvalho LR. Hábitos de saúde e fatores de risco em pacientes hipertensos. Rev Latino-Am Enfermagem 2002 mai/jun; 10(3): 1-9. de condutas, mas por uma atitude compreensiva, afetiva e efetiva nos cuidados com a saúde.

0 enfermeiro tem uma importante participação nesse processo, que envolve estilo de vida e cuidado, no sentido de facilitar a mudança de comportamento do sujeito, baseada num modelo de promoção e prevenção em saúde. Assim, ele identifica comportamentos de risco e implementa práticas de cuidados adequadas, uma vez que é o profissional que lida diretamente com o cliente na maior parte do tempo ${ }^{15}$.

Esse trabalho se restringiu a uma descrição de dados quantitativos, e, como faz parte de uma pesquisa, ele tem continuidade, de modo que estudos posteriores requerem cruzamentos dos dados e contextualização com dados qualitativos.

Enfim, essa pesquisa visa, com seus resultados, buscar nova forma de concepção do estilo de vida do cliente e criar alternativas de atenção em saúde adequadas à realidade da população.

Esse estudo teve apoio da Pró-Reitoria de Pesquisa e Pós-Graduação da Universidade Federal Fluminense (PROPP-UFF) e da Fundação de Amparo à Pesquisa do Estado do Rio de Janeiro (FAPERJ).

8. Vieira ZM, Goulart JCT, Fiamoncini RL, Galli GB. Atividade física e hipertensão. Rev Digital Buenos Aires. 2004 out; [citado outubro de 2004]; 10 (77). Disponível em: http://www.efdeportes.com

9. Organização Pan-Americana da Saúde-OPAS. Doenças crônicodegenerativas e obesidade: estratégia mundial sobre alimentação saudável, atividade física e saúde. Brasília (DF); 2003.

10. Mello FJ. Psicossomática hoje. Porto Alegre (RS): Artes Médicas; 1992.

11. Pessuto J, Carvalho EC. Fatores de risco em indivíduos com hipertensão arterial. Rev Latino-Am Enfermagem 1998 jan; 6(1): 33-9.

12. Pires ZRS, Silva MJ. Autonomia e capacidade decisória dos idosos de baixa renda: uma problemática a ser considerada na saúde do idoso. Rev Eletrônica Enfermagem Goiânia. 2001 jul/dez; [citado outubro de 2004]; 3 (2): [aprox.19 telas]. Disponível em: http:// www.fen.ufg.br/revista/revista3_2/autonomia.html

13. Smeltzer SC, Bare BG. Brunner \& Suddarth. Tratado de Enfermagem Médico-Cirúrgica. 9a ed. Rio de Janeiro (RJ): Guanabara Koogan; 2002.

14. Shoji VM, Fortaz CLM. Treinamento físico na hipertensão arterial. Rev Soc Cardiol Estado São Paulo 2000 nov/dez; 10 (6): 7-14.

15. Potter PA, Perry AG. Fundamentos de enfermagem. Rio de Janeiro (RJ): Guanabara Koogan; 2004.

16. Rente F. Comportamentos aditivos: dependência e antropologia. J Primeiro de Janeiro 2005 jul 29. 
17. Klein CH, Araújo JWG. Fumo, bebida alcoólica, migração, instrução, ocupação, agregação familiar e pressão arterial em Volta Redonda. Cad Saúde Pública 1985 abr/jun; 1 (2): 160-76.

18. Júnior MD et al. Crenças de saúde do paciente com hipertensão arterial. Rev Paul Enferm 1988 abr/mai/jun; 8 (2): 4-7.

19. Fadden MAJ, Ribeiro AV. Aspectos psicológicos e hipertensão essencial. Rev Assoc Med Bras Hipertensão 1998 jan/mar; 44 (1): 5-8.

20. Sparrenberger F, Moreira LB, Caneppele MCGL. Associação entre estresse e hipertensão. Rev Soc Bras Hipertensão 2004; 7 (3): 96-9.

21. Machado SC, Stipp MAC, Leite JL. Clientes com hipertensão arterial: perspectiva da gerência do cuidado de enfermagem. Esc Anna Nery Rev Enferm 2005 abr; 9(1): 64-71.]

22. Reich W. A função do orgasmo. São Paulo (SP): Brasiliense; 1998.

23. Teixeira ER. A crítica e a sensibilidade no processo de cuidar. Esc Anna Nery Rev Enferm 2004 dez; 8(3): 361-69.

\section{Sobre os Autores}

\section{Enéas Rangel Teixeira}

Professor Titular do Departamento de Enfermagem Médico-cirúrgica da Escola de Enfermagem Aurora de Afonso Costa/Universidade Federal Fluminense, Niterói/RJ. e-mail:psicosoma@vm.uff.br.

\section{Alinny Rodrigues Lamas}

Acadêmica de Enfermagem da Escola de Enfermagem Aurora de Afonso Costa/Universidade Federal Fluminense, Niterói/RJ. Bolsista de iniciação científica da FAPERJ.

\section{Juliana da Costa e Silva}

Acadêmica de Enfermagem da Escola de Enfermagem Aurora de Afonso Costa /Universidade Federal Fluminense, Niterói/RJ. Bolsista de Iniciação Científica da PROPP/UFF

\section{Ronivaldo Menegussi de Matos}

Acadêmico de Enfermagem da Escola de Enfermagem Aurora de Afonso Costa/Universidade Federal Fluminense, Niterói/RJ. Bolsista de iniciação científica da FAPERJ. 